\title{
PROPRIETÀ E LEGGI DI NATURA
}

\author{
CRISTIAN MARIANI (*)
}

SunTO. - La nozione di legge di natura è al centro della riflessione filosofica sulla scienza. Nel tentativo di determinare quale sia lo statuto modale di queste leggi, se esse siano necessarie (necessitarismo) oppure contingenti (contingentismo), il recente dibattito si è in larga parte incentrato sulla metafisica delle proprietà naturali. Il presente contributo si inserisce in questo dibattito, e ha come scopo quello di difendere una tesi sull'individuazione delle proprietà naturali nota come strutturalismo. Secondo lo strutturalismo, le proprietà sono esaustivamente individuate dal loro ruolo nomologico/causale, e dunque dal loro rapporto con le altre proprietà e con le leggi. Nello specifico, difendo una versione dello strutturalismo inteso come teoria delle controparti per le proprietà. Lo strutturalismo implica una forma di necessitarismo sulle leggi di natura, e dunque il presente testo può essere visto come una difesa di questa tesi.

$* * *$

ABSTRACT. - The notion of law of nature has been a major focus on the recent philosophical debate on science. In the attempt of determining what is the modal status of laws, whether they are necessary (as for necessitarianism) or contingent (as for contingentism), the recent debate has largely focused on the relationship between laws and natural properties. This contribution aims at defending a particular view on property individuation known as structuralism. According to structuralism, roughly, properties are individuated by their nomological/causal roles, and so by their relationships with the other properties and with the laws. In specific, I will defend a version of structuralism as a counterpart theory of property. Structuralism implies necessitarianism on laws of nature, and so this paper can be seen as a defense of this view as well.

(*) Università degli Studi di Milano, Dipartimento di Filosofia, Centre for Philosophy of Time, Milano, Italia; Rutgers University, USA.

Email: mariani_cristian@yahoo.it. 


\section{INTRODUZIONE}

Fra i temi più discussi e controversi nella filosofia contemporanea, quello sullo statuto della conoscenza scientifica occupa senza dubbio un posto di massimo rilievo. La scienza moderna infatti, e la fisica in modo preminente, in ragione del suo enorme successo e del suo costante progresso è divenuta il simbolo di una conoscenza tanto sofisticata quanto affidabile. In molti hanno di conseguenza affermato che i problemi che per secoli si erano ritenuti di natura squisitamente filosofica, ad esempio sulla natura del tempo e dello spazio, o sul significato della causalità, siano di fatto comprensibili solo alla luce delle nostre migliori teorie scientifiche. Posto però che la scienza moderna possa sostituirsi alla filosofia in quanto indagine sulla natura ultima del mondo naturale, resta nondimeno viva la riflessione sul senso e sui limiti di quella conoscenza, sul suo linguaggio e sulle presupposizioni metafisiche che le soggiacciono. Una filosofia o una metafisica che si dicano naturalistiche, hanno appunto come obiettivi quelli di affermare, da un lato, la fiducia nella conoscenza scientifica, e di riflettere criticamente, dall'altro, sui presupposti, il linguaggio, e la natura di quella conoscenza. Il presente saggio intende inserirsi all'interno di una tradizione ormai decennale di una metafisica naturalistica. Nello specifico, intende riflettere su una nozione fra le più centrali per la scienza, quella di legge di natura.

Attraverso le leggi, la scienza mira a fornire delle generalizzazioni dei fenomeni naturali. Alcune fra queste generalizzazioni, con il passare del tempo, possono rivelarsi false o approssimate. Ciò nondimeno, esse si presentano come universali, necessarie ed esplicative. Queste caratteristiche sono il segno distintivo di una legge di natura. Si pensi alla legge secondo cui cariche di segno opposto si attraggono. L'universalità implica che la validità della legge non è ristretta ma vale per ciascuno degli oggetti nel dominio della legge stessa. Dunque, nell'esempio, la legge implica che ogni carica è attratta da una carica di segno opposto. La necessità inoltre, preclude che la validità delle leggi sia un mero accidente. In altre parole, non è un puro caso che tutte le cariche di segno opposto si attraggano. Infine, la natura esplicativa delle leggi sta ad indicare che $\mathrm{i}$ singoli fenomeni naturali sono spiegati dalle leggi. Sperimentando due cariche di segno opposto attrarsi, possiamo cioè spiegare questo comportamento riferendoci alla legge. In assenza di una legge, il fenomeno resterebbe cioè privo di spiegazione.

La scienza, in larga misura, si occupa di fornire generalizzazioni il 
più accurate e corrette possibile. Come detto, alcune fra queste generalizzazioni si rivelano scorrette, e vengono sostituite o modificate con il progresso scientifico. Eppure, fra gli obiettivi della scienza vi è quello di giungere, idealmente, a formulare quelle leggi di natura che siano vere ed universali. Che tale obiettivo sia di fatto raggiungibile o meno, d'altronde, nulla ha a che vedere con il significato dell'impresa. Potrebbe cioè darsi che non saremo mai in grado di formulare o scoprire le vere leggi di natura - forse in ragione della loro complessità, o perché non ne avremo il tempo - ma che, ciò nonostante, tali leggi di fatto esistano. Benché la scienza abbia come compito quello di formulare le leggi di natura, essa non ha però come suo obiettivo quello di definire che cosa una legge di natura sia, o di indicare come distinguere una legge di natura da una generalizzazione meramente accidentale. Questo compito spetta senza dubbio alla riflessione filosofica. Fra i molti quesiti che la nozione di legge di natura solleva, il più storicamente discusso in filosofia è senza dubbio quello che riguarda lo statuto modale delle leggi: le leggi di natura sono da considerarsi necessarie? Se sì, di che tipo di necessità si tratta?'

In questo contributo intendo fornire una parziale risposta a questi quesiti. Nello specifico, intendo difendere una teoria sulla individuazione delle proprietà nota come strutturalismo, dalla quale deriva la necessità delle leggi di natura, cioè il necessitarismo sulle leggi. La connessione fra la tesi strutturalista e quella necessitarista - e più in generale fra proprietà e leggi - è mostrata nella sezione (1). Le sezioni (2) e (3)

1 'Necessario' ha più significati. C'è anzitutto una necessità di tipo meramente logico: la proposizione 'piove o non piove' è necessaria in questo senso. C'è poi una necessità di tipo naturale, o nomologica, che attribuiamo appunto ai fenomeni naturali: che la velocità della luce sia di circa $300 \mathrm{~km}$ al secondo è un esempio. Ovviamente però, quando ci domandiamo della necessità delle leggi di natura non possiamo riferirci ad uno di questi due sensi appena descritti. Dire che le leggi sono necessarie in senso nomologico sarebbe infatti nulla di più che un vuoto pleonasmo. Dire invece che esse sono necessarie logicamente appare troppo forte, o persino privo di senso, dal momento che la necessità logica pare derivare da principi logici (nell'esempio sopra, dal principio di bivalenza); è chiaro che nel caso delle leggi di natura non ci sono principi logici dai quali esse derivano la propria necessità. Se le leggi di natura non sono necessarie in alcuno di questi sensi, che cosa intendiamo col dire che le leggi sono necessarie? Nel domandarci se le leggi siano necessarie intendiamo riferirci ad un terzo senso che la nozione di necessità possiede, un senso che chiamiamo metafisico. Per un approfondimento, si veda Fine (2002). 
sono dedicate alla definizione delle due principali tesi sulla individuazione delle proprietà, cioè rispettivamente il quidditismo e lo strutturalismo. Nella sezione (4), infine, difendo lo strutturalismo da alcuni argomenti avanzati da Locke (2010).

\section{LO STATUTO MODALE DELL LEGGI E LE PROPRIETÀ NATURALI}

Nel recente dibattito filosofico sulle leggi di natura due questioni appaiono intimamente connesse. La prima questione riguarda lo statuto modale delle leggi, e cioè se esse siano da considerarsi necessarie oppure contingenti. Il necessitarismo ${ }^{2}$ considera le attuali leggi di natura vere in ogni mondo possibile, quindi necessarie. Il contingentismo è la negazione di questa tesi. La seconda questione concerne l'individuazione delle proprietà naturali. Secondo lo strutturalismo, ${ }^{4}$ le proprietà sono esaustivamente individuate dal loro profilo nomologico o causale, cioè dal ruolo che esse realizzano all'interno delle leggi. Il

2 Una difesa estesa del necessitarismo è Bird (2007), e più recentemente Wilson (2016).

3 Il contingentismo è stato notoriamente difeso da David Lewis (1973; 2009), e David Armstrong (1983). Lewis (1973) ha sviluppato la cosiddetta 'regularity theory of laws', che fa riferimento anche a Ramsey (1928). Armstrong (1983) (si veda anche: Tooley (1977), e Dretske (1977)) ha invece promosso la 'universals theory of laws'. Non è oggetto del presente contributo quello di discutere in dettaglio queste teorie. Piuttosto, la mia attenzione sarà rivolta alla relazione fra il dibattito sull'individuazione delle proprietà e quello fra necessitarismo e contingentismo. In tal senso, le differenze fra le posizioni di Lewis e di Armstrong — che pure sono significative - possono essere lasciate fra parentesi.

4 Lo strutturalismo è stato promosso, fra gli altri, da Shoemaker (1998), Black (2000), Hawthorne (2001), Bird (2007), e Kistler (2002). È utile notare che lo strutturalismo come tesi concernente l'individuazione delle proprietà va distinto dalla tesi strutturalista in filosofia della scienza (si veda: French (2014), e Ladyman \& Ross (2007). Il secondo è, più o meno esplicitamente, una forma di anti-realismo delle proprietà (si veda Esfeld (2014) per una discussione più estesa). Il primo invece, che è l'interesse di questo contributo, non nega l'esistenza delle proprietà ma afferma che la loro identificazione è una questione in qualche misura olistica, o o appunto strutturale. Questo tipo di confusione è in parte il motivo per cui molti autori preferiscono il termine 'causal essentialism' (Wang 2016) o 'dispositional essentialism' (e.g., Bird 2007). Il motivo per cui preferisco mantenere l'appellativo strutturalismo è che ritengo preferibile indicare una discriminante con la tesi disposizionalista; dopo tutto, lo strutturalismo non sembra costretto ad 
quidditismo5 - dal latino quidditas, ad indicare la natura intrinseca di una proprietà ${ }^{6}$ - è la negazione dello strutturalismo. Secondo i quidditisti la natura ultima di una proprietà trascende il ruolo che essa svolge nelle leggi, e non è dunque esaurita da esso. Che il profilo nomologico/causale non esaurisca la natura di una proprietà implica inoltre che la connessione fra proprietà e leggi sia contingente. Per fare un esempio, secondo i quidditisti la proprietà 'essere carico positivamente', se posseduta, non è necessariamente tale da implicare che l'oggetto che la possiede sia attratto da un oggetto carico negativamente. Il contingentismo sulle leggi di natura implica dunque il quidditismo, così come il necessitarismo implica lo strutturalismo.

Si possono distinguere due tipi di necessitarismo, debole e forte. ${ }^{7}$ Secondo il necessitarismo forte, le attuali leggi di natura sono necessarie nel senso di vere in tutti i mondi possibili. ${ }^{8}$ In altre parole, questa forma di necessitarismo stabilisce che possibilità nomologica e possibilità metafisica sono coestensive. Il necessitarismo debole mantiene invece questa distinzione, stabilendo però che lo spazio della possibilità metafisica concerne solo quei mondi con proprietà e leggi diverse da quelle attuali; le attuali leggi di natura sono però considerate necessarie, nel senso di vere in tutti i mondi che contengono le attuali proprietà.?

accettare una metafisica di disposizioni, mentre il disposizionalista deve in qualche misura accettare la tesi strutturalista (Locke (2012, pp. 353-354) concorda con questa considerazione).

5 Il quidditismo è stato recentemente difeso da molti autori. Una lista non esaustiva include: Lewis (2009), Schaffer (2005), Locke (2012), Smith (2016), e Hildebrand (2016).

6 Più precisamente, per la tarda scolastica il termine quidditas indicava la natura essenziale di un ente. La quidditas, assieme alla baecceitas completava l'identificazione di un oggetto. Nel linguaggio contemporaneo, il termine è invece usato per indicare il corrispettivo per le proprietà di ciò che l'baecceitas è per gli individui, vale a dire, appunto, la loro natura o essenza.

7 Seguo Bird (2007) nel definire questa visione necessitarismo forte, piuttosto che necessitarismo modale (come fanno Schaffer (2005) e Wilson (2016)), perché ritengo che la distinzione fra forte e debole permetta intuitivamente di cogliere la differenza nell'estensione dello spazio modale ammesso dalle due tesi. Ciò nondimeno, è corretto ricordare che in un certo senso la forma 'forte' sia maggiormente modally laden, come anche Hildebrand (2016) fa notare.

8 Bird (2007), Wilson (2016).

9 Shoemaker (1998). 
Possiamo domandarci quali motivazioni abbiamo per preferire una tesi piuttosto che l'altra. Il principale argomento a favore del contingentismo fa appello al combinatorialismo, un principio che molti ritengono governare i nostri ragionamenti sulla possibilità e necessità ${ }^{10} \mathrm{Il}$ principio si basa a sua volta sulla stretta connessione fra concepibile e possibile, secondo cui una proposizione $p$ è possibile se e solo se $p$ è concepibile. Il combinatorialismo sancisce che possiamo costruire scenari possibili (cioè concepibili) ricombinando entità distinte. Per il contingentista ciò vuol dire che, dal momento che possiamo concepire un mondo possibile in cui cariche di segno opposto si respingano, tramite il combinatorialismo possiamo costruire un mondo possibile in cui ciò di fatto accade. L'esistenza di un tale mondo possibile prova che il necessitarismo è falso. Il necessitarista può negare che proprietà e leggi siano entità distinte, o può negare il principio che connette possibilità e concepibilità. Entrambe queste strade comportano però una eccessiva revisione dei nostri concetti. ${ }^{11}$ Un'altra strada è quella di abbracciare una forma di necessitarismo debole, e affermare che gli scenari concepiti sono di fatto scenari in cui le leggi e le proprietà sono diverse da quelle attuali (nella letteratura tali proprietà e leggi sono definite aliene, poiché riguardano mondi che non sono quello attuale). Ciò comporta una restrizione al combinatorialismo, ${ }^{12}$ ma in molti ritengono che tale restrizione sia non solo indipendentemente motivata, ma anche e soprattutto tale da non costringerci ad una revisione delle nostre teorie sulla modalità.

Per ciò che riguarda invece gli argomenti a favore del necessitarismo, essi sono spesso argomenti rivolti contro il quidditismo. Possiamo distinguerli in due tipi: epistemologici e metodologici. ${ }^{13} \mathrm{I}$ primi mirano a stabilire

10 L' argomento basato sul principio di ricombinazione è presente in Armstrong (1983), Lewis (2009), e più di recente in Schaffer (2005).

11 Schaffer (2005) argomenta in esteso che il rifiuto del contingentismo implica il rifiuto delle nostre teorie standard sui controfattuali, sulle proposizioni, concepibilità, e in generale sulla modalità. Ritengo però che la forza di queste considerazioni sia molto maggiore in riferimento al necessitarismo forte (ad esempio, Wilson (2016) nella sua difesa del necessitarismo forte ammette che tale revisione sia inevitabile), laddove non è chiaro se e in che misura sia convincente nel caso del necessitarismo debole.

12 Hawthorne (2001), ad esempio, presente un principio di ricombinazione ristretto che di fatto è compatibile con il necessitarismo debole. Dirò qualcosa su questo principio nella sezione (3).

13 Va inoltre notate che vi è una terza categoria di argomenti contro il 
che il quidditismo è una teoria epistemicamente infondata, dal momento che afferma l'esistenza di entità che sono per loro natura di fatto inconoscibili. ${ }^{14}$ Nelle prossime sezioni intendo però concentrare l'attenzione sugli argomenti di tipo metodologico. In particolare, intendo argomentare che le risposte fornite dai quidditisti — in particolare da Locke (2010) — non sono in grado di fornire una difesa accettabile. La mia conclusione sarà dunque che lo strutturalismo è una teoria da preferire al quidditismo.

\section{IL QUIDDITISMO}

Il termine quidditas viene usato nel linguaggio filosofico contemporaneo riferito alle proprietà per indicarne la natura intrinseca, l'essenza, o l'baecceitas. Benché gli autori non concordino del tutto sulla definizione esatta, è utile individuare quello che è un punto di accordo fra tutti coloro che hanno discusso la nozione di quidditas e la tesi del quidditismo. Indipendentemente dalla definizione, affinché il quidditismo possa supportare la tesi contingentista sulle leggi naturali, il seguente principio deve essere vero:

Realizzazione Quiddistica (RQ):

Per almeno un ruolo nomologico $R$ realizzato da una proprietà $P$,

è possibile che $R$ sia realizzato senza essere realizzato da $P .{ }^{15}$

Il principio stabilisce chiaramente che la connessione fra proprietà e ruoli è contingente, pur rimanendo silente su che cosa individui le proprietà, e sul senso in cui la loro natura trascenda la realizzazione di un ruolo nomologico. Locke (2010) argomenta a ragione che ogni quidditista debba accettare (RQ), e che al contrario lo strutturalismo sia la negazione di questo principio.

Con questa definizione preliminare possiamo ora comprendere l'argomento metodologico contro il quidditismo. Nella sua forma più

quidditismo, che è più di natura squisitamente metafisica. Un chiaro esempio è Black (2000), il quale argomenta che il quidditismo implica una moltiplicazione del numero di mondi possibile tale che questi sono infiniti non numerabili.

14 Shoemaker (1998).

15 Per una discussione estesa su (RQ), si veda Locke (2010). 
efficace, l'argomento è discusso da Hawthorne (2001) con il nome di argomento della parsimonia:

«La miglior considerazione a favore dell'idea che il profilo causale di una proprietà ne esaurisca la sua natura passa per l'idea che "non abbiamo bisogno di quidditas per dare senso al mondo'. [Consideriamo] la carica negativa. Tutta la conoscenza scientifica riguardo alla carica negativa, è conoscenza del ruolo causale che essa realizza. La scienza non sembra offrire alcuna concezione della carica negativa come qualche cosa che sia al di là di 'quella cosa che realizza quel certo ruolo'. Se ci fosse una quidditas che sia, per così dire, un 'tappabuchi', ciò sarebbe un qualcosa a cui la scienza non avrebbe alcun diretto accesso cognitivo, salvo come referente dell'espressione 'la quidditas che realizza il ruolo di carica negativa'. Perché dunque invocare ciò di cui non si ha bisogno? A meno che considerazioni logiche ci forzino a supporre che le proprietà siano individuate da qualcosa che trascende il ruolo causale, perché porre l'esistenza di misteriose quidditas?» (368-369, traduzione mia).

Il passaggio contiene due considerazioni di estrema rilevanza. Anzitutto, Hawthorne fa notare che le proprietà sono « nulla al di fuori del loro ruolo » - in altre parole, Hawthorne sembra suggerire che il modo corretto di intendere la negazione del quidditismo sia di identificare i ruoli con le proprietà. Il secondo punto è di carattere più propositivo: in quanto 'tappabuchi', le quidditas sono entità misteriose e non necessarie, di cui la scienza non ha alcun bisogno.

In risposta ad Hawthorne, recentemente Locke (2010) ha proposto una forma di quidditismo che sarebbe in grado di raggirare l'argomento della parsimonia. Locke distingue due diverse tesi che il quidditista potrebbe difendere:

\section{Quidditismo Stravagante:}

Le proprietà sono individuate dalle quidditas.

Quidditismo Austero:

Le proprietà sono individuate dall'identità numerica (non vi sono quidditas)

Secondo Locke, la confusione fra le due tesi deriva dall'aver ritenuto che il principio RQ richieda di porre l'esistenza delle quidditas, cosa per nulla scontata. Locke fa poi notare - credo in modo corretto - che l'argomento della parsimonia ha effetto solo contro il quidditismo strava- 
gante, mentre non ha presa su quello austero dal momento che quest'ultimo semplicemente nega l'esistenza di quidditas. Posto che Locke abbia ragione su questo punto, resta da domandarsi che cosa voglia dire che le proprietà siano individuate meramente dall'identità numerica.

Locke invita a pensare al quidditismo austero come ad una teoria primitivista dell'identità delle proprietà attraverso mondi possibili. Al contrario, il quidditismo stravagante è una teoria delle controparti per le proprietà. Per teoria delle controparti — in riferimento alla più nota teoria di Lewis degli individui ${ }^{16}$ - si intende una teoria secondo cui la rappresentazione modale de re dipende da qualcosa di diverso dalla mera identità numerica. Per Lewis, ad esempio, l'identità fra individui attraverso i mondi possibili dipende appunto dalle controparti di questi individui. Le controparti permettono dunque di fissare il valore di verità di proposizioni modali attraverso i mondi, senza che gli individui siano concepiti come entità che abitano più mondi (si noti che per Lewis ciò è di estrema importanza dal momento che egli è un realista sui mondi possibili). Ebbene, così come la teoria lewisiana degli individui è una teoria modale delle controparti, il quidditismo stravagante è un teoria dell'individuazione delle proprietà attraverso delle controparti. Il 'qualche cosa che trascende il ruolo', nelle parole di Hawthorne, cioè la quidditas, è appunto la controparte. Locke conclude che non abbiamo ragione di difendere una teoria delle controparti per le proprietà, e invita dunque al quidditismo austero, il quale per altro permette di rispondere all'obiezione metodologica di Hawthorne.

Avendo chiarito qual è il cuore della tesi quidditista, nella prossima sezione introduco quella che ritengo la migliore versione dello strutturalismo, per poi valutare e rigettare alcuni argomenti contro quest'ultimo nella sezione (5).

\section{LO STRUTTURALISMO}

Kistler (2002) definisce lo strutturalismo come la tesi per cui «l'identità di un universale è interamente determinata dalle relazioni nomologiche con le altre proprietà » (p. 57, traduzione mia). Che cosa sono queste relazioni? Una buona strategia per definire le relazioni strutturali,

16 Lewis (1986). 
o ruoli realizzati dalle proprietà, è fornita da Hawthorne (2001), il quale propone di usare la tecnica cosiddetta della ramseyficazione (dal filosofo Ramsey). Questa tecnica, adottata ampiamente anche da Lewis, permette di definire i concetti senza riferirsi direttamente ad essi.

$\mathrm{Nel}$ caso delle proprietà, un esempio concreto di ramseyficazione è il seguente. Si considerino cinque proprietà naturali — chiamiamole A, B, C, D ed E. Supponiamo ora che queste proprietà siano connesse dalle seguenti quattro leggi di natura (utilizzo la notazione di Armstrong per indicare una legge): ${ }^{17}$

1. $\mathrm{A} N \mathrm{~B}$;

2. $\mathrm{A} N \mathrm{NC}$

3. $\mathrm{B} N \mathrm{D}$;

4. DNE.

Supponiamo ora, per semplicità, che queste quattro leggi e queste cinque proprietà costituiscano la totalità della leggi e delle proprietà. La ramseyficazione consiste ora nel formare una proposizione, che chiamiamo proposizione-Ramsey, congiungendo le cinque leggi, e sostituendo le proprietà con variabili quantificate esistenzialmente. Nell'esempio, abbiamo dunque:

$$
\begin{array}{llllllll}
\mathrm{F}^{1} & \mathrm{~F}^{2} & \mathrm{~F}^{3} & \mathrm{~F}^{4} & \mathrm{~F}^{5}\left(\mathrm{~F}^{1} \mathcal{N} \mathrm{F}^{2}\right. & \mathrm{F}^{1} \mathcal{N} \mathrm{F}^{3} & \mathrm{~F}^{3} \mathcal{N} \mathrm{F}^{4} & \left.\mathrm{~F}^{4} \mathcal{N} \mathrm{F}^{5}\right)
\end{array}
$$

Infine, per definire un certo ruolo realizzato da una proprietà si prenda A come esempio - eliminiamo semplicemente il quantificatore esistenziale davanti alla variabile che sostituisce $A$ (cioè $\mathrm{F}^{1}$ ). Si noti che questa forma di strutturalismo è in grado, almeno parzialmente, di rispondere all'obiezione basata sul principio di ricombinazione. L'idea, in estrema sintesi, è quella di limitare il principio alle possibili ricombinazioni di ruoli strutturali (che, di nuovo, definiamo attraverso la ramseyficazione), e come conseguenza si ha che ad ogni mondo possibile corrisponderà una diversa proposizione-Ramsey. ${ }^{18}$

17 Seguo Hawthorne (2001) nell'uso della teoria e della notazione di Armstrong (1983) delle leggi di natura. Per questi, la relazione $N$ di necessitazione connette due universali, ed è dunque espressione di una legge di natura.

18 Per dettagli, si veda: Hawthorne, 2001, 371. 
La maggiore problematica con questa forma di strutturalismo, come lo stesso Hawthorne fa notare, è connessa alla possibilità di ruoli strutturalmente simmetrici (Hawthorne, 2001, 374-5). Si prenda la seguente struttura nomologica, composta da quattro proprietà $\mathrm{A}$, $\mathrm{B}, \mathrm{C}$, e $\mathrm{D}$, e da tre leggi, $\mathrm{A} \mathcal{N} \mathrm{C}, \mathrm{B} \mathcal{N} \mathrm{C},(\mathrm{A} \& \mathrm{~B}) \mathcal{N} \mathrm{D}$. Si noti che il ruolo di $\mathrm{A}$ e $\mathrm{B}$ è di fatto indistinguibile, poiché entrambe le proprietà, se da sole necessitano $\mathrm{C}$, e se congiunte necessitano $\mathrm{D}$. Ciò nondimeno, esse sono distinte, poiché solo se congiunte possono necessitare $\mathrm{D}$. I loro ruoli sono appunto simmetrici. La conclusione è che non vi è un modo di costruire una proposizione-Ramsey da questa possibilità. La risposta di Hawthorne (p. 375) è che si consideri lo strutturalismo una teoria de re delle rappresentazioni modali. Si prenda ad esempio il ruolo $\mathrm{R}^{\mathrm{A}}$ realizzato dalla proprietà $\mathrm{A}$. Lo strutturalismo come teoria de re è la tesi per cui una certa possibilità $w$ rappresenta una proprietà $\mathrm{P}$ in una certa maniera se e solo se nella possibilità $w$ la proprietà $\mathrm{P}$ realizza il ruolo $\mathrm{R}^{\mathrm{P}}$ in quella maniera. I ruoli simmetrici sono preservati come genuine possibilità, ma solo all'interno di un certo mondo. Tali possibilità però non rappresentano mondi genuinamente possibili, ma solo differenze quiddistiche la cui esistenza lo strutturalista nega.

\section{L'IDENTITÀ DEI RUOLI FRA MONDI E LE CONTROPARTI DELLE PROPRIETÀ}

Locke e Hawthorne condividono dunque l'idea che il modo corretto di pensare allo strutturalismo sia come una teoria delle controparti per le proprietà. In questa sezione valuto e rigetto due argomenti di Locke (2010) contro lo strutturalismo così inteso.

Nel primo argomento Locke comincia col considerare che, se lo strutturalismo è da intendere come teoria delle controparti, non sembrano esserci motivazioni sufficienti per non avere una teoria delle controparti anche per i ruoli, generando così un apparente regresso:

«[...] che cosa rende una proprietà controparte di un'altra? Supponiamo sia il condividere un ruolo. Possiamo allora domandare: la proprietà $\mathrm{P}$ e la sua controparte $\mathrm{P} *$ condividono un ruolo in virtù del fatto che ci sia un ruolo che entrambi $\mathrm{P}$ e $\mathrm{P} *$ possiedono? Oppure esse condividono i ruoli in virtù del condividere i ruoli delle controparti? Presumibilmente, lo strutturalista vuole mantenere che il condividere ruoli sia una questione di genuina identità fra ruoli. Ma per- 
ché fermarsi qui? Perché non dire che condividere ruoli è una questione di condividere ruoli delle controparti? Ovviamente, una volta che una tale teoria delle controparti è stata fornita, saremmo di nuovo di fronte ad una nuova questione. Ma l'ascesa alle teorie delle controparti deve finire da qualche parte. Altrimenti, non ci sarebbero fatti a proposito di rappresentazioni modali de re neppure al più basso dei livelli.» (358)

Non è chiaro se e in che misura, tuttavia, lo strutturalista debba essere mosso da considerazioni di questo genere dal momento che per lui, chiaramente, l'ascesa alle teorie delle controparti' termina al livello dei ruoli. Per lo strutturalista, infatti, è chiaro che i ruoli siano primitivi, e dunque che siano identici attraverso i mondi.

Locke considera poi un ulteriore argomento contro lo strutturalismo come teoria delle controparti, che è di fatto una sorta di rafforzamento del primo argomento. Esso si basa sul seguente principio metodologico introdotto dallo stesso Locke (p. 358):

\section{Principio metodologico sulla teoria delle controparti}

Dobbiamo arrestare l'ascesa alle teorie delle controparti quando raggiungiamo il livello in cui non ci sono ragioni per adottare una teoria delle controparti.

Da questo principio, senza dubbio estremamente intuitivo, secondo Locke seguirebbe che, a meno che non ci siano ragioni per adottare una teoria delle controparti al livello delle proprietà, dovremmo arrestarci al livello degli individui, e adottare una teoria dell'identità primitiva per le proprietà.

In risposta, si consideri il seguente principio metodologico:

Principio metodologico sulla teoria delle controparti *

Dobbiamo arrestare l'ascesa alle teorie delle controparti quando non c'è un ulteriore livello nel quale abbia senso adottare una teoria delle controparti.

Questo secondo principio, non meno intuitivo del precedente, sembra piuttosto favorire lo strutturalismo. Possiamo infatti domandarci quale sarebbe il livello al di là di quello dei ruoli. Similarità fra proprietà ha senso rispetto alla condivisione di ruoli, così come similarità fra individui (come nella teoria di Lewis) ha senso rispetto alla condivi- 
sione di proprietà. Che cosa sarebbe, invece, similarità fra ruoli? Presumibilmente, di nuovo proprietà strutturali, cioè condivisione di ruoli. La morale da trarre dal confronto fra questi due principi, e dal fatto che essi suggeriscano diverse conclusioni, è che, quando si arriva ad un tale livello di astrazione come nel presente caso, sembra improprio potersi affidare alla metodologia nella speranza che possa dettarci una tesi metafisica piuttosto che l'altra.

\section{CONCLUSIONI}

Ho mostrato come il dibattito sullo statuto modale delle leggi di natura - se le leggi siano cioè da considerarsi contingenti o necessarie - sia strettamente connesso alla teoria attraverso cui individuiamo le proprietà naturali che nelle leggi compaiono. Ho poi introdotto il quidditismo e lo strutturalismo, le due principali tesi sull'individuazione delle proprietà, illustrando quelle che ritengo le formulazioni migliori; il quidditismo austero di Locke (2010), e lo strutturalismo come teoria delle controparti per le proprietà introdotto da Hawthorne (2001). Ho infine difeso lo strutturalismo da alcune argomentazioni avanzate da Locke (2010). In conclusione, ritengo che lo strutturalismo sia una tesi da preferire al quidditismo, e che dunque il necessitarismo (almeno di tipo debole) sulle leggi ne consegua.

\section{RINGRAZIAMENTI}

Desidero ringraziare David Ingram, Max Kistler, Benjamin Neeser, Jonathan Schaffer, Jonathan Tallant e Giuliano Torrengo, per aver discusso pazientemente con me parti di questo testo, e per averne migliorato il contenuto in maniera sostanziale. Desidero poi ringraziare Lorenzo Capitanio, Giuseppe Moro e Nicola Alessio Sarracco, per la lettura e la revisione di una precedente versione di questo testo e per $\mathrm{i}$ loro utilissimi consigli. Qualsiasi errore rimasto è di responsabilità esclusiva del sottoscritto. Un ringraziamento speciale va infine a Mauro D'Ariano, per avermi dato la possibilità di contribuire a questo volume, e più in generale per aver condiviso con me, nell'arco degli ultimi cinque anni, la sua enorme passione e dedizione per la fisica e per la filosofia della scienza. 


\section{BIBLIOGRAFIA}

Armstrong D.M., (1983): What is a Law of Nature, Cambridge; Cambridge University Press.

(1989): A combinatorial theory of Possibility, Cambridge; Cambridge University Press.

Bird A., (2007): Nature's Metaphysics. Laws and Properties, Oxford; Oxford University Press.

Black R., (2000): 'Against Quidditism', Australasian Journal of Philosophy, 78(1): 87-104.

Curtis B., (2016): 'Lewisian quidditism, humility, and diffidence', Philosophical Studies, 173:3081-3099.

Dretske F., (1977): 'Laws of nature', Philosophy of Science, 44, 248-68.

Ellis B. (1999): 'Causal Powers and Laws of Nature', in H. Sankey (ed.), Causation and Laws of Nature, Dordrecht: Kluwer: 19-34.

(2001): Scientific Essentialism, Cambridge: Cambridge University Press.

Esfeld M., (2014): 'Quantum Humeanism, or: Physicalism without Properties', Philosophical Quarterly 64 (256):453-470.

Fine, (2002): 'Varieties of Necessity', in Tamar Szabo Gendler \& John Hawthorne (eds.), Conceivability and Possibility, OUP, pp. 253-281 (2002)

French S., (2014): The Structure of the World, Oxford: Oxford University Press.

Hawthorne J., (2001): 'Causal Structuralism', Phil Perspective, 8:122-139.

Hildebrand T., (2016): 'Two types of Quidditism', Australasian Journal of Philosophy, 93(3): 516-532.

Kelly A., (2013): 'Ramseyan Humility, Scepticism and Grasp', Philosophical Studies, 164(3): 705-726.

Kistler M., (2002): 'The Causal Criterion of Reality and the Necessity of Laws of Nature', in Metaphysica, 1(3): pp. 57-86.

Ladyman J. \& Ross D., (2007): Every Thing Must Go: Metaphysics Naturalized, Oxford: Oxford University Press.

Lewis D.K., (1973): Counterfactuals, Oxford: Blackwell. (1986): On the Plurality of Worlds, Oxford: Blackwell. (2009): 'Ramseyan Humility', in D. Braddon-Mitchell \& R. Nold (Eds.), Conceptual analysis and philosophical naturalism, Cambridge (MA): MIT Press.

Locke D., (2009): Quidditism, PhD dissertation.

- (2012): 'Quidditism without quiddities', Philosophical Studies, 160: 345-363.

Schaffer J., (2005): 'Quiddistic knowledge', Philosopbical Studies, 130(3):461-497.

Shoemaker S., (1998): 'Causal and metaphysical necessity', Pacific Philosophical Quarterly, 79:59-77.

Smith D., (2016): 'Quid Quidditism Est?', Erkenntniss, 81: 237-257.

Tooley, (1977): 'The nature of laws', Canadian Journal of Philosophy, 7, 667-98.

Wang, (2016): 'The Nature of Properties: Causal Essentialism and Quidditism', Philosophy Compass 11 (3):168-176.

Wilson A., (2016): 'Schaffer on laws of nature', Philosophical Studies 164 (3):653-667. 